KUDRYAVTSEV Pavel Gennadievich, D.Sc., Professor of HIT (Israel), Academician of International Academy of Sciences for Ecology and Human Safety and Russian Academy of Natural Sciences, author of more than 150 publications including «Nanomaterials based on soluble silicates» (in cooperation with O.Figovsky) and 30 inventions; 52 Golomb Street, РОВ 305 Holon 5810201, Израиль, 23100, e-mail:pgkudr89@gmail.com;

FIGOVSKY Oleg Lvovich, Full Member of European Academy of Sciences, Foreign Member of REA and RAASN, Editor-in-Chief of Journals SITA (Israel), OCJ and ICMS (USA), Director R\&D of INRC Polymate (Israel) and Nanotech Industries, Inc. (USA); Chairman of the UNESCO chair «Green Chemistry»; President of Israel Association of Inventors; Laureate of the Golden Angel Prize, Polymate INRC; P.O.Box 73, Migdal Ha'Emeq, Израиль, 10550, e-mail: figovsky@gmail.com

\title{
QUASI-HOMOGENOUS APPROXIMATION FOR DESCRIPTION OF THE PROPERTIES OF DISPERSED SYSTEMS. THE BASIC APPROACHES TO MODEL HARDENING PROCESSES IN NANODISPERSED SILICA SYSTEMS.
}

\section{PART II. THE HARDENING PROCESSES FROM THE STANDPOINT OF STATISTICAL PHYSICS}

The paper deals with possibilities to use quasi-homogenous approximation for discription of properties of dispersed systems. The authors applied statistical polymer method based on consideration of average structures of all possible macromolecules of the same weight. The equiations which allow evaluating many additive parameters of macromolecules and the systems with them were deduced. Statistical polymer method makes it possible to model branched, cross-linked macromolecules and the systems with them which are in equilibrium or non-equilibrium state. Fractal analysis of statistical polymer allows modeling different types of random fractal and other objects examined with the mehods of fractal theory. The method of fractal polymer can be also applied not only to polymers but also to composites, gels, associates in polar liquids and other packaged systems. There is also a description of the states of colloid solutions of silica oxide from the point of view of statistical physics. This approach is based on the idea that colloid solution of silica dioxide - sol of silica dioxide - consists of enormous number of interacting particles which are always in move. The paper is devoted to the research of ideal system of colliding but not interacting particles of sol. The analysis of behavior of silica sol was performed according to distribution Maxwell-Boltzmann and free path length was calculated. Using this data the number of the particles which can overcome the potential barrier in collision was calculated. To model kinetics of sol-gel transition different approaches were studied.

Key words: quasi-homogenous approximation, dispersed systems, statistic polymer method, formation of crosslinkings, fractal method, colloid solution, silica, sol-gel transition, free path length.

DOI: dx.doi.org/10.15828/2075-8545-2015-7-2-62-84 


\section{References:}

1. Kudryavtsev P., Figovsky O. Nanomaterials based on soluble silicates, ISBN 978-3659-63556-4, LAP Lambert Academic Publishing, 2014, 241 p.

2. Kudryavtsev P., Figovsky $O$. Nanomaterialy na osnove rastvorimyh silikatov [Nanomaterials based on soluble silicates], ISBN 978-3-659-58361-2, LAP Lambert Academic Publishing, 2014, 155 p. (In Russian).

3. Morachevskij A.P. Fizicheskaja himija - poverhnostnye javlenija i dispersnye sistemy [Physical chemistry - surface phenomena and dispersed systems], SaintPetersburg, 2011. (In Russian).

4. Lao L., Orsinger E. Hyperbolic and fractional hyperbolic Brownian motion, Stochastics: An International Journal of Probablty and Stochastics Processes, p. 505$522,2007$.

5. Bondarev B.V., Kalashnikov N.P., Spirin G.G. Kurs obshhej fiziki: v 3 kn. Kniga 3. Statisticheskaja fizika. [Course of general physics: in 3 volumes. Volume 3. Statistical physics. Substance structure]. Moscow, Jurajt, 2013, 369 p.

6. Lifshic E.M., Pitaevskij L.P. Statisticheskaja fizika. Chast' 2. Teorija kondensirovannogo sostojanija. («Teoreticheskaja fizika», tom IX) [Statistical physics. Part 2. The theory of condensed state. («Theoretical physics», volume IX). Moscow, Fizmatlit, 2004, 496 p.

7. Schmidt M. Simulations of Systems with Colloidal Particles, in: Simulations of Systems with Colloidal Particles, ISBN: 0-8247-0323-5, edited by Borowko M., New York, Basel, Marcel Dekker, inc., 2000, pp. 745-773.

8. Segrè P.N., Behrend O.P., Pusey P.N. Short-time Brownian motion in colloidal suspensions: Experiment and simulation, PhysRevE., 1995, Vol. 52 5, pp. 507005083, Doi: 10.1103/PhysRevE.52.5070, http://link.aps.org/doi/ 10.1103/PhysRevE.52.5070.

9. Sanyal Subrata, Sood Ajay K. Brownian dynamics simulation of dense binary colloidal mixtures. I. Structural evolution and dynamics, Phys. Rev. E, Vol. 52, 4, pp. 4154-4167, 1995, doi: 10.1103/PhysRevE.52.4154, http://link.aps.org/ doi/10.1103/PhysRevE.52.4154.

10. Sanyal Subrata, Sood Ajay K. Brownian dynamics simulation of dense binary colloidal mixtures. II. Translational and bond-orientational order, Phys. Rev. E, Vol. 52, 4, pp. 4168-4178, 1995, doi: 10.1103/PhysRevE.52.4168, http://link. aps.org/doi/10.1103/PhysRevE.52.4168.

11. Lowe C.P., Frenkel D. Short-time dynamics of colloidal suspensions, Phys. Rev. E, Vol. 54, 3, pp. 2704-2713, 1996, doi: 10.1103/PhysRevE.54.2704, http://link. aps.org/doi/10.1103/PhysRevE. 54.2704.

12. Hagen M.H.J., Pagonabarraga I., Lowe C.P., Frenkel D. Algebraic Decay of Velocity Fluctuations in a Confined Fluid, Phys. Rev. Lett., Vol. 78, 19, pp. 3785-3788, 1997, doi: 10.1103/PhysRevLett.78.3785, http://link.aps.org/doi/10.1103/ PhysRevLett.78.3785. 
13. Groot R.D., Warren P.B. Dissipative particle dynamics: Bridging the gap between atomistic and mesoscopic simulation, J. Chem. Phys. Vol. 107, 10, p. 4423, 1997, http://dx.doi.org/10.1063/1.474784.

14. Oberholzer M.R., Wagner N.J., Lenhoff A.M. Grand canonical Brownian dynamics simulation of colloidal adsorption, J. Chem. Phys. 107, 9157 (1997); http:// dx.doi.org/10.1063/1.475207.

15. Zahn K., Méndez-Alcaraz J.M., Maret G. Hydrodynamic Interactions May Enhance the Self-Diffusion of Colloidal Particles, Phys. Rev. Lett., Vol. 79, 1, pp. 175178, 1997, doi: 10.1103/PhysRevLett.79.175, http://link.aps.org/doi/10.1103/ PhysRevLett.79.175.

16. Sunil Kumar P. B., Rao M. Novel Monte Carlo Approach to the Dynamics of Fluids: Single-Particle Diffusion, Correlation Functions, and Phase Ordering of Binary Fluids, Phys. Rev. Lett., Vol. 77, 6, pp. 1067-1070, 1996, doi: 10.1103/PhysRevLett.77.1067, http://link.aps.org/doi/10.1103/PhysRevLett.77.1067.

17. Laradji M. Toxvaerd S., Mouritsen O.G. Molecular Dynamics Simulation of Spinodal Decomposition in Three-Dimensional Binary Fluids, Phys. Rev. Lett., Vol. 77, 11, pp. 2253-2256, 1996, doi: 10.1103/PhysRevLett.77.2253, http://link.aps. org/doi/10.1103/PhysRevLett.77.2253.

18. Löwen H. Brownian dynamics of hard spherocylinders, Phys. Rev. E, Vol. 50, 2, pp. 1232-1242, 1994, doi: 10.1103/PhysRevE.50.1232, http://link.aps.org/ doi/10.1103/PhysRevE.50.1232.

19. Löwen $H$. Anisotropic self-diffusion in colloidal nematic phases, Phys. Rev. E, Vol. 59, 2, pp. 1989-1995, 1999, doi: 10.1103/PhysRevE.59.1989, http://link. aps.org/doi/10.1103/PhysRevE.59.1989.

20. Kirchhoff Th., Löwen H., Klein R. Dynamical correlations in suspensions of charged rodlike macromolecules, Phys. Rev. E, Vol. 53, 5, pp. 5011-5022, 1996, doi: 10.1103/PhysRevE.53.5011, http://link.aps.org/doi/10.1103/PhysRevE.53.5011.

21. Romm F., Figovsky O. Statistical polymer method: Modeling of macromolecules and aggregates with branching and crosslinking, formed in random processes, Discrete Dynamics in Nature and Society Volume 2 (1998), Issue 3, P. 203-208, http://dx.doi.org/10.1155/S1026022698000181.

22. Romm F., Figovsky O. Modeling of Mechanical Properties of Polymeric Systems with Branching/Crosslinking, Particularly Their Mechanical Resistence and Stability. Macromolecular Theory and Simulations Volume 11, Issue 1, pp. 93-101, January 2002.

23. Romm F., Karchevsky V., Figovsky O. Combined monte carlo/thermodynamic model of formation of microporous aggregate structure like silica from quaternary ammonium silicate solutions. Journal of Surfactants and Detergents(IF 1.515), 2000, Vol.3 (4), pp. 475-481, Springer. http://onlinelibrary.wiley.com/doi/10.1002/1521-3919\% 2820020101\% 2911:1\% 3C93::AIDMATS93\% 3E3.0.CO;2-F/abstract. 
24. Ponomarenko A.T., Figovsky O., Shevchenko V.G. Multifunctional Polymer Composites for «Intellectual» Structures: Present State, Problems, Future. Journal Advanced Materials Research, 2008, Vol.740 (47), pp.81-84, Trans Tech.

25. Figovsky O.L., Beilin D.A., Ponomarev A.N.Successful implementation of nanotechnologies in building materials. Nanotehnologii v stroitel'stve $=$ Nanotechnologies in Construction. 2012, Vol. 4, no. 3, pp.6-21. Available at: http://nanobuild.ru/ en_EN/. (In Russian).

26. Kudryavtsev P.G., Figovsky O.L. Nanostructured materials, production and application in construction. Nanotehnologii v stroitel'stve = Nanotechnologies in Construction. 2014, Vol. 6, no. 6, pp. 27-45. DOI: dx.doi.org/10.15828/2075-85452014-6-6-27-45.

27. Kudryavtsev P., Figovsky O. Quasi-homogenous approximation for description of the properties of dispersed systems. The basic approaches to model hardening processes in nanodispersed silica systems. Nanotehnologii v stroitel'stve = Nanotechnologies in Construction. 2015, Vol. 7, no. 1, pp. 29-54. DOI: dx.doi. org/10.15828/2075-8545-2015-7-1-29-54.

Dear Colleagues!

THE REFERENCE TO THIS PAPER HAS THE FOLLOWING CITATION FORMAT:

Kudryavtsev P.G., Figovsky O.L. Quasi-homogenous approximation for description of the properties of dispersed systems. The basic approaches to model hardening processes in nanodispersed silica systems. Part II. The hardening processes from the standpoint of statistical physics. Nanotehnologii v stroitel'stve = Nanotechnologies in Construction. 2015, Vol. 7, no. 2, pp. 62-84. DOI: dx.doi. org/10.15828/2075-8545-2015-7-2-62-84.

\section{Contact information}

Figovsky 0.

figovsky@gmail.com 
КУДРЯВЦЕВ Павел Геннадьевич, профессор, D.Sc. Холонский технологический институт (Израиль), академик МАНЭБ и РАЕН, автор 150 научных работ, в том числе «Наноматериалы на основе растворимых силикатов» (в соавторстве с О. Фиговским), имеет более 30 изобретений; 52 Golomb Street, POВ 305 Holon 5810201, Израиль, 23100, e-mail: pgkudr89@gmail.com;

ФИГОВСКИЙ Олег Львович, действительный член Европейской академии наук, иностранный член РИА и РAACH, главный редактор журналов SITA, OCJ и RPCS, директор компании «Nanotech Industries, Inc.», Калифорния, США, директор Международного нанотехнологического исследовательского центра «Рolymate» (Израиль), зав. кафедрой ЮНЕСКО «Зелёная химия», президент Израильской Ассоциации Изобретателей, Лауреат Golden Angel Prize, Polymate INRC; P.O.Box 73, Migdal Ha’Emeq, Израиль, 10550, e-mail: figovsky@gmail.com

\section{КВАЗИГОМОГЕННОЕ ПРИБЛИЖЕНИЕ ДЛЯ ОПИСАНИЯ СВОЙСТВ ДИСПЕРСНЫХ СИСТЕМ. ОСНОВНЫЕ ПОДХОДЫ К МОДЕЛИРОВАНИЮ ПРОЦЕССОВ ОТВЕРЖДЕНИЯ В ДИСПЕРСНЫХ СИЛИКАТНЫХ СИСТЕМАХ.}

\section{ЧАСТЬ ІІ. ПРОЦЕССЫ ОТВЕРХДЕНИЯ С ТОЧКИ ЗРЕНИЯ СТАТИСТИЧЕСКОЙ ФИЗИКИ}

В этой статье мы рассматривали возможности применения квазигомогенного приближения для описания свойств дисперсных систем. Мы использовали статистический полимерный метод на основе рассмотрения усредненных структур всех возможных макромолекул одинакового веса. Выведены уравнения, позволяющие оценить многие аддитивные параметры макромолекул и содержащих их систем. Статистический полимерный метод позволяет моделировать разветвленные, сшитые макромолекулы и содержащие их системы, находящиеся в состоянии равновесия или в неравновесном состоянии. Фрактальное рассмотрение статистического полимера позволяет моделировать различные виды случайного фрактала и других объектов, изучаемых методами фрактальной теории. Способ статистического полимера применим не только к полимерам, но также и к композитам, гелям, ассоциатам в полярных жидкостях и другим агрегативным системам. В данной работе было описано состояние коллоидных растворов оксида кремния с точки зрения статистической физики. Такой подход основан на идее, состоящей в том, что коллоидный раствор диоксида кремния - золь диоксида кремния - состоит из очень большого числа взаимодействующих друг с другом частиц, находя- 
щихся в непрерывном движении. Она посвящена изучению идеализированной системы сталкивающихся, но не взаимодействующих частиц золя. Был проведен анализ поведения золя кремнезема с точки зрения распределения Максвелла-Больцмана, и была рассчитана средняя длина свободного пробега коллоидных частиц. На основании этих данных было рассчитано количество частиц, способных преодолеть потенциальный барьер при столкновении. Для моделирования кинетики золь-гель перехода были рассмотрены различные подходы.

Ключевые слова: квазигомогенное приближение, дисперсные системы, статистический полимерный метод, образование сшивок, фрактальный метод, коллоидный раствор, кремнезоль, золь-гель переход, длина свободного пробега.

DOI: dx.doi.org/10.15828/2075-8545-2015-7-2-62-84

\section{6. Описание состояния коллоидных растворов оксида кремния с точки зрения статистической физики.}

В последнее десятилетие резко возрос интерес к исследованиям в области применения нанотехнологий в строительных материалах, поскольку результат таких исследований может стать основой внедрения в практику принципиально новых материалов, обладающих уникальными физико-механическими и химическими характеристиками [25]. Однако создание подобных материалов невозможно без детального описания поведения первичных наночастиц в процессе формирования наноструктурированного материала [26]. Попытка такого подхода была предпринята в первой части настоящей статьи [27].

Этот раздел основывается на представлении, заключающемся в том, что коллоидный раствор оксида кремния - кремнезоль, состоит из очень большого числа взаимодействующих друг с другом частиц, которые находятся в непрерывном движении $[1,2]$.

Он посвящен изучению идеализированной системы сталкивающих-

ся, но не взаимодействующих частиц золя. Для упрощения подхода рассмотрим идеальную систему монодисперсных коллоидных частиц. Поскольку частицы золя значительно больше молекул растворителя, в котором они находятся, то их взаимодействиями с молекулами рас- 
творителя можно пренебречь и рассматривать эту систему как взвесь частиц золя в некой непрерывной среде - континууме. Частицы золя большую часть времени движутся свободно и не сталкиваются друг с другом, такое их движение носит название броуновское движение. Они взаимодействуют только при столкновениях, в результате которых скорость и направление движения каждой из сталкивающихся частиц изменяется. Поэтому траектория движения частиц в золе представляет собой ломаную линию [3].

Теория броуновского движения нашла широкое применение не только для описания случайного движения частицы в жидкости, но и для решения целого ряда прикладных задач статистической механики. Этой теории подчиняются случайные тепловые колебания высокоточных механических и электрических измерительных устройств. Кинетические уравнения, полученные в теории броуновского движения, используются для анализа точности работы различных систем управления. Они позволяют рассчитать случайные ошибки, возникающие при управлении техническими устройствами, и провести оптимизацию их параметров. Законами броуновского движения определяется случайное движение электронов, вызывающее шумы в электрических цепях. Диэлектрические потери в диэлектриках объясняются случайными движениями молекул-диполей, составляющих диэлектрик. Случайные движения ионов в растворах электролитов увеличивают их электрическое сопротивление. Случайное броуновское движение производит частотную диаграмму, которая может быть использована для предсказания вещей, включающих большие количества данных и статистики. Броуновские случайные движения коллоидных частиц приводят к получению фрактальных агрегатов. Также броуновское движение применимо для получения фракталов из фракталов [4, 7].

К такой системе могут быть применены, с определенным допущением, законы идеальных газов. Рассмотрим систему из $\mathrm{N}$ частиц, заполняющих некоторый объем пространства V. Распределение частиц в пространстве описывается посредством функции:

$$
C_{n}=C_{n}(t, \vec{r})
$$

зависящей от времени и координат. Эта функция называется счетной концентрацией и определяется следующим образом. Разобьем про- 
странство, заполненное частицами золя, на малые части и рассмотрим одну из этих частей, объем которой обозначим $d V$. Объем $d V$ называется физически бесконечно малым, если число $d N$ частиц внутри него существенно меньше полного числа $N$ частиц в объеме $V$, но при этом во много раз больше единицы: $1 d N N$. Положение объема $d V$ в пространстве можно задать при помощи радиус-вектора $\vec{r}$ одной из его точек. Счетной концентрацией частиц в данном месте пространства называется отношение числа $d N$ частиц в объеме $d V$ к величине этого объема:

$$
C_{n}(t, \vec{r})=\frac{d N}{d V}
$$

Так как при своем движении частицы могут входить в объем $d V$ и выходить из него, то число частиц $d N$ в этом объеме, строго говоря, будет изменяться со временем [5]. Поэтому концентрация является функцией не только радиус-вектора $\vec{r}$, но и времени $t$. Путем интегрирования уравнения (II.2) получим число $N$, равное количеству частиц в объеме $V$ :

$$
N=\int_{V} C_{n}(t, \vec{r}) d V
$$

Когда частицы распределены по объему сосуда в среднем равномерно, их концентрация всюду одинакова, и формула (II.3) принимает вид

$$
N=C_{n} \cdot V \text {. }
$$

Физический смысл счетной концентрации может быть определен как число частиц в единичном объеме. В самом деле, положив $V=1 \mathrm{~m}^{3}$, получим $C_{n}=N$.

Рассмотрим вероятность того, что одна произвольно выбранная частица золя в момент времени $t$ окажется в объеме $d V$ :

$$
d W=\frac{d N}{N} .
$$

При этом можно получить величину плотности вероятности:

$$
w(t, \vec{r})=\frac{d W}{d V} .
$$


Плотность вероятности еще называется функцией распределения частиц золя в пространстве. Эта функция связана со счетной концентрацией золя следующим простым соотношением:

$$
C_{n}(t, \vec{r})=N w(t, \vec{r})
$$

Для случая монодисперсных частиц можно легко получить выражение для массовой концентрации частиц золя в единице объема:

$$
C(t, \vec{r})=m_{p} C_{n}(t, \vec{r})
$$

где $\boldsymbol{m}_{p}$ - масса одной частицы золя. Для сферических частиц золей диаметром $\boldsymbol{d}_{p}$, при плотности вещества золя $\rho$, она равна:

$$
m_{p}=V_{p} \rho=\frac{\pi d_{p}^{3}}{6} \rho \text {. }
$$

В случае полидисперсной системы необходимо ввести новую функцию для счетной концентрации, которая еще зависит и от массы частиц соответствующего размера $-C_{n m}\left(t, m_{p}, \vec{r}\right)$. Соответственно, выражение для массовой концентрации примет вид:

$$
C_{n}(t, \vec{r})=\int_{0}^{\infty} \varphi\left(m_{p}\right) C_{n m}\left(t, m_{p}, \vec{r}\right) d m_{p},
$$

где $\varphi\left(m_{p}\right)$ - функция распределения частиц по массе в золе. Однако для упрощения описания и анализа системы частиц золя мы остановимся на упрощенном случае монодисперсного золя.

Для описания микроскопического состояния золя используют функцию

$$
f=f(t, r, \overrightarrow{0}) \text {. }
$$

Эта функция зависит от времени $t$, радиус-вектора $\vec{r}$ и вектора скорости $\vec{v}$ и называется функцией распределения. Она определяется следующим образом. Рассмотрим частицы, которые в момент времени $t$ оказались в объеме $d V$ при радиус-векторе $\vec{r}$. Число таких частиц $-d N$. Движение каждой частицы характеризуется некоторым вектором скорости $\vec{v}_{i}$, где $i$ - номер частицы [6]. 
Построим воображаемую прямоугольную систему координат, на осях которой будем откладывать значения проекций $v_{x}, v_{y} u v_{z}$ вектора скорости каждой из частиц в этом объеме. Пространство, образованное при помощи этой системы координат, называется пространством скоростей. Выделим в пространстве скоростей небольшой (физически бесконечно малый) объем, величину которого обозначим $d^{3} v$. Положение этого объема в пространстве скоростей зададим вектором $\vec{v}$, который начинается в начале координат и заканчивается в какой-нибудь точке внутри объема $d^{3} v$. Скорости некоторых частиц из числа $d N$ заканчиваются в этом объеме: $\overrightarrow{v_{1}} \in d^{3} v$. Пусть их число равно $d N^{\prime}$. Причем это число удовлетворяет неравенствам $1<<d N^{\prime}<<d N$. Функция распределения (II.11) определяется как отношение числа частиц $d N^{\prime}$ к величине произведения объемов $d V$ и $d^{3} v$ :

$$
f(t, \hat{r}, \hat{v})=\frac{d N^{\prime}}{d V d^{3} v} .
$$

Из этого определения следует, что число частиц $d N^{\prime}$, которые в момент времени $t$ оказались в объеме $d V$ при радиус-векторе $\vec{r}$, а их векторы скоростей заканчиваются в объеме $d^{3} v$ при векторе $\vec{v}$, можно вычислить, если известна функция распределения (II.11). Разделив переменные и интегрируя уравнение (II.12) по объему, а затем разделив его на $d V$, и учитывая, что интегрирование производится по пространству скоростей, получим равенство:

$$
C_{n}(t, \vec{r})=\int f(t, \vec{r}, \vec{v}) d^{\mathrm{a}} v
$$

Это уравнение связывает концентрацию частиц с их функцией распределения. Величину элементарного объема $d^{3} v$ в пространстве скоростей можно представить в виде произведения дифференциалов разложения вектора скорости $\vec{v}$ по осям координат, что превращает интеграл (II.13) в тройной интеграл:

$$
C_{n}(t, x, y, z)=\int_{-\infty}^{+\infty} \int_{-\infty}^{+\infty} \int_{-\infty}^{+\infty} f\left(t, x, y, z, v_{x}, v_{y}, v_{z}\right) d v_{x} d v_{y} d v_{z},
$$

где интегрирование производится по всем возможным значениям величин . 
Согласно определению (II.5) можно определить вероятность того, что одна произвольно взятая частица золя в момент времени $t$ находится в объеме $d V$, а ее скорость - в объеме $d^{3} v$ пространства скоростей. Coответствующая плотность вероятности определяется как отношение

$$
w(t, \hat{r}, \hat{v})=\frac{d W}{d V d^{3} v} .
$$

Из формулы (II.12) следует, что плотность вероятности связана с функцией распределения (II.11) соотношением

$$
f(t, \vec{r}, \vec{v})=N w(t, \vec{r}, \vec{v}) .
$$

Распределение частиц по скоростям называется изотропным, если функция распределения зависит только от модуля $v$ вектора скорости $\vec{v}$ и не зависит от его направления: $f=f(t, \vec{r}, v)$. При таком распределении частиц по скоростям потоки частиц, летящих в различных направлениях, одинаковы. Другими словами, все направления движения частиц в пространстве равноценны. В таком случае средняя скорость направленного движения частиц, очевидно, должна быть равна нулю: $\vec{u}=0$. Это означает, что направленное движение частиц отсутствует. Такая ситуация характерна для тех случаев, когда в системе отсутствует перемешивание и когда частицы золя достаточно малы, и эффектом седиментации частиц, под действием гравитации, можно пренебречь.

Важным фактором, определяющим поведение золя, являются средние скорости движения частиц золя. Для их определения положим, что $d N$ есть число частиц в объеме $d V$. Найдем среднее значение $\bar{v}$ модуля $v$ вектора скорости частиц. Для этого следует просуммировать модули скоростей всех частиц, находящихся в объеме $d V$, и полученную сумму разделить на число частиц:

$$
\bar{v}=\frac{1}{d N} \sum_{i} v_{i},
$$

где индекс $i$ обозначает номера частиц, находящихся в объеме $d V$.

Так как объем $d^{3} v$ в пространстве скоростей достаточно мал для тех частиц, скорости которых заканчиваются в этом объеме, можно положить $\vec{v}_{i}=\vec{v}$ при $\vec{v}_{i} \in d^{3} v$, где $\vec{v}-$ произвольный вектор, заканчивающий- 
ся в объеме $d^{3} v$. Поэтому сумма модулей скоростей этих частиц будет равна произведению модуля $v$ скорости одной частицы на их число $d N^{\prime}$ :

$$
v d N^{\prime}=v f(t, \vec{r}, \vec{v}) d V d^{3} v .
$$

Сумма модулей скоростей всех частиц в объеме $d V$ будет равна интегралу по пространству скоростей от этого выражения:

$$
\sum_{i} v_{i}=d V \int v f(t, \vec{r}, \vec{v}) d^{3} v
$$

Подстановка этой суммы в формулу (II.17) с учетом (II.2) дает

$$
\bar{v}=\frac{1}{C_{n}} \int v f(t, \vec{r}, \vec{v}) d^{3} v .
$$

Собственно, это уравнение представляет собой момент 1-го порядка функции трехмерного распределения вероятностей. Аналогичным образом можно вывести формулы для моментов других порядков функ-

ции распределения вероятностей. Например, для средних значений $\overline{\vec{v}}$ и $\overline{v^{2}}$ вектора скорости и квадрата его модуля будем иметь следующие формулы:

$$
\begin{aligned}
& \overline{\vec{v}} \equiv \vec{u}(t, \vec{r})=\frac{1}{C_{n}} \int \vec{v} f(t, \vec{r}, \vec{v}) d^{3} v, \\
& \overline{v^{2}}=\frac{1}{C_{n}} \int v^{2} f(t, \vec{r}, \vec{v}) d^{3} v .
\end{aligned}
$$

В общем случае вектор $\vec{u}$ средней скорости частиц не равен нулю и является функцией от времени и радиус-вектора. Это означает, что в данный момент времени в данном месте пространства вся масса золя перемещается как целое в определенном направлении. Образно выражаясь, золь течет или его перемешивают в реакционном сосуде. При решении некоторых задач удобно считать, что частицы золя участвуют сразу в двух движениях: хаотическом тепловом и направленном. Направленное движение характеризуется вектором средней скорости $\vec{u}$.

Заметим, что интегрирование в формулах (II.20) - (II.22) производится по пространству скоростей при заданных значениях $t$ и $\vec{r}$. Поэто- 
му полученные после интегрирования выражения в общем случае будут представлять собой некоторые функции от времени и координат.

Величина

$$
v_{R M S} \equiv\langle v\rangle=\sqrt{\overline{v^{2}}}
$$

называется средней квадратичной скоростью частицы.

\section{7. Анализ поведения кремнезоля с точки зрения распределения Максвелла-Больцмана}

Рассмотрим кремнезоль, находящийся в состоянии термодинамического квазиравновесия. Функцию (II.11), описывающую распределение частиц золя в пространстве и по скоростям, можно найти при помощи канонического распределения Гиббса. Для этого следует рассматривать коллоидный раствор как ансамбль, каждая из частиц которого является одной из его систем. При этом параметр в каноническом распределении Гиббса следует понимать как совокупность пространственных координат $x, y, z$ и проекций $v_{x}, v_{y}, v_{z}$ вектора скорости частицы:

$$
x \equiv\left\{x, y, z, v_{x}, v_{y}, v_{z}\right\} \equiv\{r, 0\} .
$$

Согласно закону Гиббса плотность вероятности для одной из частиц в приближении идеального газа для системы, находящейся в состоянии термодинамического равновесия или близкого к равновесию состояния, будет иметь вид

$$
\begin{aligned}
& w(\vec{r}, \hat{0})=v \exp (-\beta \varepsilon(\vec{r}, \hat{v})), \\
& \text { где } \beta=(k T)^{-1}-\text { обратная температура, а } \\
& \varepsilon(\vec{r}, \overrightarrow{0})=\frac{1}{2} m v^{2}+U(\vec{r})
\end{aligned}
$$

- та часть энергии частицы, которая зависит от ее координат и скорости, т.е. сумма кинетической энергии поступательного движения частицы и ее потенциальной энергии. Это уравнение справедливо, если в пространстве имеется внешнее поле консервативной силы (например, на частицы действует сила тяжести). Кроме энергии, описанной в урав- 
нении (II.25), частица обладает также внутренней энергией, которая в данной ситуации не оказывает существенного влияния на свойства ансамбля частиц с точки зрения их взаимодействия друг с другом.

Зная плотность вероятности (II.24), функцию распределения найдем по формуле (II.16):

$$
f(\vec{r}, \vec{v})=N v \exp \left\{-\beta\left(\frac{1}{2} m v^{2}+U(\vec{r})\right)\right\} .
$$

Функции (II.24) и (II.26) носят названия распределений Максвелла-Больцмана. Одной из характерных особенностей этих функций является то, что они зависят только от модуля вектора скорости и не зависят от его направления в пространстве. Иначе говоря, эти функции описывают изотропное распределение частиц по скоростям.

Функцию распределения (II.25) удобно представить в виде произведения двух функций:

$$
f(\vec{r}, \vec{t})=n(\vec{r}) \cdot w(\vec{v}) .
$$

Первая из них представляет собой концентрацию частиц:

$$
n(\vec{r})=n_{0} \exp (-\beta U(\vec{r})) .
$$

Вторая является плотностью вероятности:

$$
w(\hat{v})=\mathbf{A} \exp \left(-\alpha v^{2}\right),
$$

где $\mathrm{A}$ - нормировочная постоянная, а $\alpha$ равна

$$
\alpha=\frac{\beta m}{2}=\frac{m}{2 k T} \text {. }
$$

Постоянные $n_{0}$ и А связаны соотношением:

$$
n_{0} A=N v \text {. }
$$

Функцию (II.28) называют распределением Больцмана, а функцию (II.29) - распределением Максвелла. Первая описывает распределение частиц в пространстве, а вторая - их распределение по скоростям. 
Подстановка функции (II.27) в равенство (II.13) приводит к условию нормировки для функции (II.29):

$$
\int w(\vec{v}) d^{3} v=1 .
$$

Разложив интеграл (II.31) по осям вектора скоростей частиц и использовав выражение для интеграла Пуассона, найдем нормировочную постоянную:

$$
A=\left(\frac{\alpha}{\pi}\right)^{\frac{3}{2}} \text {. }
$$

Используя полученное выражение (II.32) для нормировочной постоянной, запишем распределение Максвелла (II.29) следующим образом:

$$
w(\hat{v})=\left(\frac{\alpha}{\pi}\right)^{\frac{3}{2}} \exp \left(-\alpha v^{2}\right) .
$$

Эту функцию можно представить в виде произведения трех функций:

$$
w(\vec{v})=g\left(v_{x}\right) g\left(v_{y}\right) g\left(v_{z}\right),
$$

где

$$
g\left(v_{x}\right)=\left(\frac{\alpha}{\pi}\right)^{\frac{1}{2}} \exp \left(-\alpha v_{x}^{2}\right)
$$

есть так называемая функция Гаусса. Вычислим среднюю скорость $\mathrm{u}$ направленного движения частиц золя по формуле для первого момента функции распределения, которая для функции распределения Максвелла-Больцмана принимает вид:

$$
\vec{u}=\overline{\vec{v}}=\int \vec{v} w(\vec{v}) d^{3} v .
$$

Используя функцию (II.34), будем иметь для среднего значения проекции вектора скорости на ось $x$ следующее выражение:

$$
u_{x} \equiv \bar{v}_{x}=\int_{-\infty}^{+\infty} v_{x} g\left(v_{x}\right) d v_{x} \int_{-\infty}^{+\infty} g\left(v_{x}\right) d v_{x} \int_{-\infty}^{+\infty} g\left(v_{x}\right) d v_{x} .
$$


Как и следовало ожидать, для изотропного распределения частиц по скоростям это выражение равняется нулю, потому что равен нулю интеграл от нечетной функции $v_{x} g\left(v_{x}\right)$. Таким образом, средняя скорость направленного движения частиц, скорости которых распределены по закону Максвелла, равна нулю: $\vec{u}=\mathbf{0}$.

Отсюда среднее значение квадрата проекции вектора скорости на ось $x$ составит:

$$
\overline{v_{x}^{2}}=\int v_{x}^{2} w(\vec{v}) d^{3} v .
$$

Если разложить этот интеграл по осям пространства скоростей частиц и подставить в него выражение (II.34), получим:

$$
\overline{v_{x}^{2}}=\int_{-\infty}^{+\infty} v_{x}^{2} g\left(v_{x}\right) d v_{x} \int_{-\infty}^{+\infty} g\left(v_{x}\right) d v_{x} \int_{-\infty}^{+\infty} g\left(v_{x}\right) d v_{x} .
$$

Интегрирование этого выражения дает формулу:

$$
\overline{v_{x}^{2}}=\frac{k T}{m} .
$$

В силу свойств случайных величин, когда среднее значение суммы двух случайных величин равно сумме средних значений этих величин, среднее значение квадрата модуля скорости будет равно:

$$
\overline{v^{2}}=\overline{\left(v_{x}^{2}+v_{y}^{2}+v_{z}^{2}\right)}=\overline{v_{x}^{2}}+\overline{v_{y}^{2}}+\overline{v_{z}^{2}} .
$$

Равновесная функция распределения (II.26) зависит от модуля вектора скорости и не зависит от его направления, т.е. описывает изотропное распределение частиц по скоростям, все направления которых равноценны. Поэтому среднее значение квадрата проекции вектора скорости на любую ось не зависит от направления этой оси в пространстве:

$$
\overline{v_{x}^{2}}=\overline{v_{y}^{2}}=\overline{v_{z}^{2}},
$$

при этом

$$
\overline{v^{2}}=3 \overline{v_{x}^{2}},
$$


а с учетом формулы (II.37) будем иметь:

$$
\overline{v^{2}}=\frac{3 k T}{m} \text {. }
$$

Используя определение (II.23), найдем среднюю квадратичную скорость частицы:

$$
\langle v\rangle=\sqrt{\frac{3 k T}{m}} .
$$

В силу того, что функция распределения Максвелла (II.29) зависит только от модуля вектора скорости и не зависит от его направления, в связи с этим на поверхности сферы радиуса $v$ в пространстве скоростей эта функция всюду принимает одно и то же значение. Число $d N^{\prime}$ частиц в объеме $d V$, модули скоростей которых принадлежат интервалу $(v, v+$ $d v)$, можно найти по формуле (II.12). Для этого подставим в эту формулу функцию (II.27) и объем

$$
d^{3} v=4 \pi v^{2} d v
$$

сферического слоя

$$
d N^{\prime}=n w(\vec{v}) d V \cdot \mathbf{4} \pi v^{2} d v .
$$

Величина

$$
d N=n d V
$$

есть число частиц в объеме $d V$. По определению отношение

$$
\frac{d N^{\prime}}{d N}=w(\vec{v}) 4 \pi v^{2} d v
$$

есть вероятность того, что одна из частиц имеет скорость, модуль которой лежит в интервале от $v$ до $v+d v$.

Введем функцию $F=F(v)$, зависящую от модуля вектора скорости, при помощи соотношения

$$
F(v) d v \equiv w(\hat{v}) 4 \pi v^{2} d v
$$


Используя выражение (II.34), будем иметь

$$
F(v)=4 \pi\left(\frac{\alpha}{\pi}\right)^{\frac{3}{2}} v^{2} \exp \left(-\alpha v^{2}\right)
$$

Зависимость (II.42) называют функцией Максвелла. При $v=0$ функция (II.42) равна нулю: $\mathrm{F}(0)=0$. При значении $v_{b}$ модуля скорости, которое называется наиболее вероятной скоростью частицы, функция Максвелла имеет максимум. В интервале $\left(0, v_{b}\right)$ она монотонно возрастает, а в интервале $\left(v_{b}, \infty\right)$ монотонно убывает, стремясь к нулю при $v \rightarrow \infty$.

Так как выражение $F(v) d v$ представляет собой вероятность, соответственно интеграл от этого выражения равен единице:

$$
\int_{0}^{m} F(v) d v=1 .
$$

Физический смысл функции Максвелла для золя можно пояснить следующим образом. Согласно определению вероятности выражение $F(v) d v$ есть доля частиц, модули скоростей которых лежат в интервале $(v, v+d v)$. При этом относительное количество частиц, скорости которых лежат в интервале от $v_{1}$ до $v_{2}$, будет выражать интегралом

$$
\frac{N\left\{v \in\left[v_{1}, v_{2}\right]\right\}}{N}=\int_{v_{1}}^{v_{2}} F(v) d v,
$$

где $N$ - полное число рассматриваемых частиц, $N\left\{v \in\left[v_{1}, v_{2}\right]\right\}-$ число частиц, модули скоростей которых лежат в интервале $\left[v_{1}, v_{2}\right]$. Найдем наиболее вероятную скорость частиц $v_{b}$. Согласно необходимому условию экстремума функции при этом значении производная функции $F=F(v)$ обращается в нуль. Приравняв нулю производную по параметру $v$, от выражения (II.42) найдем, что искомое значение наиболее вероятной скорости частиц равно:

$$
v_{b}=\frac{1}{\sqrt{\alpha}}=\sqrt{\frac{2 k T}{m}} .
$$

Подставив это значение в формулу (II.41), получим максимальное значение функции Максвелла

$$
F_{\max }=F\left(v_{b}\right)=\frac{\mathbf{4}}{e} \sqrt{\frac{\alpha}{\pi}}=\frac{4}{e} \sqrt{\frac{m}{2 \pi k T}} .
$$


Функция Максвелла (II.42) содержит в себе в качестве параметра величину $\alpha$, которая согласно формуле (II.30) зависит от температуры золя. Поэтому функция Максвелла изменяется, когда происходит изменение температуры золя. Также происходит изменение в распределении частиц по скоростям. Наиболее вероятная скорость частиц (II.44) увеличивается при возрастании температуры, тогда как максимальное значение (II.45) функции Максвелла с ростом температуры уменьшается. При этом вид графической зависимости для функции Максвелла при возрастании температуры видоизменяется так, что максимум кривой смещается вправо (в сторону больших скоростей) и становится ниже, но площадь под кривой при этом остается равной единице.

Проанализируем, как изменяется с температурой распределение частиц по скоростям. С этой целью выберем некоторое произвольное значение скорости $v_{0}$. Относительные количества частиц $\left.N\left(v<v_{0}\right)\right|_{N}$ и $N\left(v>v_{0}\right) /_{N}$ со скоростями, соответственно, меньшими и большими, чем $v_{0}$, выражаются интегралами от функции Максвелла:

$$
\frac{N\left(v<v_{0}\right)}{N}=\int_{0}^{v_{0}} F(v) d v
$$

и

$$
\frac{N\left(v>v_{0}\right)}{N}=\int_{v_{0}}^{\infty} F(v) d v .
$$

Нетрудно увидеть, что с ростом температуры количество $N\left(v<v_{0}\right)$ частиц со скоростями $v<v_{0}$ монотонно уменьшается, а количество $N\left(v>v_{0}\right)$ частиц со скоростями $v>v_{0}$ увеличивается. Это свойство позволяет рассчитать количество частиц со скоростями выше определенного уровня и, соответственно, определить количество частиц, способных взаимодействовать между собой или с другими объектами при необходимости преодоления определенных потенциальных барьеров.

Коллоидные частицы дополнительно испытывают удары от окружающих атомов или молекул растворителя. Это приводит к дополнительному осложнению броуновской динамики в коллоидных суспензиях. Изучение динамики является сложной задачей, как, конечно, в первую очередь должно быть понято для равновесного состояния си- 
стемы. Обычно известна кратковременная динамика, которая, однако, регулирует свойства системы, но при этом интересным являются свойства системы в течение длительного времени.

В работе [8] было смоделировано и сравнено с экспериментами броуновское движение для коротких промежутков времени. Для плотных бинарных коллоидных смесей были смоделированы методами броуновской динамики динамика и структурная эволюция [9], трансляционный и ориентационный в определенном направлении порядок [10]. Динамика коротких промежутков времени была исследована через автокорреляционные функции скорости [11], и был обнаружен распад флуктуации скорости в замкнутой жидкости [12]. В работе [13] была сделана попытка преодолеть разрыв между атомистическим и мезоскопическим моделированием динамики диссипативных частиц. Коллоидную адсорбцию моделировали с использованием методов броуновской динамики [14]. При этом было показано, что гидродинамические силы (как правило, это силы трения) оказались в состоянии увеличить самодиффузию коллоидных частиц [15]. С использованием метода Монте-Карло был предложен подход к динамике жидкостей [16]. Моделировался спинодальный распад в бинарных жидкостях с образованием коллоидных дисперсий [17]. Броуновское движение моделировалось для жестких сфероидов в изотропной [18] и в жидкокристаллической фазе [19]. Были смоделированы динамические корреляции в суспензии заряженных палочковидных частиц [20]. В работах [21, 24] построена модель макромолекул и агрегатов с разветвлением и сшивкой, образованная в результате случайных процессов. Метод броуновской динамики был использован для моделирования механических свойств (механической стойкости и стабильности) в полимерных системах, характеризующихся значительным разветвлением и высокой степенью сшивки $[22,24]$. Сочетание метода Монте-Карло и термодинамической модели позволило смоделировать формирование микропористой структуры агрегата кремнезема, получаемого из силикатных растворов четвертичного аммониевого основания [23, 24].

Первую часть статьи "Основные подходы к моделированию процессов отверждения в нанодисперсных силикатных систелах" Кудрявцева П.Г и Фиговского О.Л. читайте в №1/2015 года Интернет-журнала "Нанотехнологии в строительстве", а части 3 и 4 - в №3/2015 и 4/2015 Интернет-журнала «Нанотехнологии в строительстве». 


\section{Библиографический список}

1. Kudryavtsev P., Figovsky O. Nanomaterials based on soluble silicates, ISBN 978-3659-63556-4, LAP Lambert Academic Publishing, 2014, 241 p.

2. Кудрявцев П., Фиговский О. Наноматериалы на основе растворимых силикатов. ISBN 978-3-659-58361-2. - LAP Lambert Academic Publishing. - 2014. - c.155.

3. Морачевский А.П. Физическая химия - поверхностные явления и дисперсные системы. - СПб., 2011.

4. Lao L., Orsinger E. Hyperbolic and fractional hyperbolic Brownian motion, Stochastics: An International Journal of Probablty and Stochastics Processes, p. 505-522, 2007.

5. Бондарев Б.В., Калашников Н.П., Спирин Г.Г. Курс общей физики: в 3 кн. - Книга 3. Статистическая физика. Строение вещества. - М.: Юрайт, 2013. - 369 с.

6. Лифшии E.M., Питаевский Л.П. Статистическая физика. Часть 2. Теория конденсированного состояния // Теоретическая физика. - М.: Физматлит, 2004. Tom IX. $-496 \mathrm{c}$.

7. Schmidt $M$. Simulations of Systems with Colloidal Particles, in: Simulations of Systems with Colloidal Particles, ISBN: 0-8247-0323-5, edited by Borowko M., New York, Basel, Marcel Dekker, inc., 2000, pp. 745-773.

8. Segrè P.N., Behrend O.P., Pusey P. N. Short-time Brownian motion in colloidal suspensions: Experiment and simulation, PhysRevE., 1995, Vol. 52 5, pp. 50705083, Doi: 10.1103/PhysRevE.52.5070, http://link.aps.org/doi/10.1103/PhysRevE.52.5070.

9. Sanyal Subrata, Sood Ajay K. Brownian dynamics simulation of dense binary colloidal mixtures. I. Structural evolution and dynamics, Phys. Rev. E, Vol. 52, 4, pp. 4154-4167, 1995, doi: 10.1103/PhysRevE.52.4154, http://link.aps.org/ doi/10.1103/PhysRevE.52.4154.

10. Sanyal Subrata, Sood Ajay K. Brownian dynamics simulation of dense binary colloidal mixtures. II. Translational and bond-orientational order, Phys. Rev. E, Vol. 52, 4, pp. 4168-4178, 1995, doi: 10.1103/PhysRevE.52.4168, http://link.aps. org/doi/10.1103/PhysRevE.52.4168.

11. Lowe C.P., Frenkel D. Short-time dynamics of colloidal suspensions, Phys. Rev. E, Vol. 54, 3, pp. 2704-2713, 1996, doi: 10.1103/PhysRevE.54.2704, http://link. aps.org/doi/10.1103/PhysRevE. 54.2704.

12. Hagen M.H.J., Pagonabarraga I., Lowe C.P., Frenkel D. Algebraic Decay of Velocity Fluctuations in a Confined Fluid, Phys. Rev. Lett., Vol. 78, 19, pp. 3785-3788, 1997, doi: 10.1103/PhysRevLett.78.3785, http://link.aps.org/doi/10.1103/ PhysRevLett.78.3785.

13. Groot R.D., Warren P.B. Dissipative particle dynamics: Bridging the gap between atomistic and mesoscopic simulation, J. Chem. Phys. Vol. 107, 10, p. 4423, 1997, http://dx.doi.org/10.1063/ 1.474784. 
14. Oberholzer M.R., Wagner N.J., Lenhoff A.M. Grand canonical Brownian dynamics simulation of colloidal adsorption, J. Chem. Phys. 107, 9157 (1997); http:// dx.doi.org/10.1063/1.475207.

15. Zahn K., Méndez-Alcaraz J.M., Maret G. Hydrodynamic Interactions May Enhance the Self-Diffusion of Colloidal Particles, Phys. Rev. Lett., Vol. 79, 1, pp. 175178, 1997, doi: 10.1103/PhysRevLett.79.175, http://link.aps.org/doi/10.1103/ PhysRevLett.79.175.

16. Sunil Kumar P. B., Rao M. Novel Monte Carlo Approach to the Dynamics of Fluids: Single-Particle Diffusion, Correlation Functions, and Phase Ordering of Binary Fluids, Phys. Rev. Lett., Vol. 77, 6, pp. 1067-1070, 1996, doi: 10.1103/PhysRevLett.77.1067, http://link.aps.org/doi/10.1103/PhysRevLett.77.1067.

17. Laradji M. Toxvaerd S., Mouritsen O.G. Molecular Dynamics Simulation of Spinodal Decomposition in Three-Dimensional Binary Fluids, Phys. Rev. Lett., Vol. 77, 11, pp. 2253-2256, 1996, doi: 10.1103/PhysRevLett.77.2253, http://link. aps.org/doi/10.1103/PhysRevLett.77.2253.

18. Löwen $H$. Brownian dynamics of hard spherocylinders, Phys. Rev. E, Vol. 50, 2, pp. 1232-1242, 1994, doi: 10.1103/PhysRevE.50.1232, http://link.aps.org/ doi/10.1103/PhysRevE.50.1232.

19. Löwen $H$. Anisotropic self-diffusion in colloidal nematic phases, Phys. Rev. E, Vol. 59, 2, pp. 1989-1995, 1999, doi: 10.1103/PhysRevE.59.1989, http://link. aps.org/doi/10.1103/PhysRevE.59.1989.

20. Kirchhoff Th., Löwen H., Klein R. Dynamical correlations in suspensions of charged rodlike macromolecules, Phys. Rev. E, Vol. 53, 5, pp. 5011-5022, 1996, doi: 10.1103/PhysRevE.53.5011, http://link.aps.org/doi/10.1103/PhysRevE.53.5011.

21. Romm F., Figovsky O. Statistical polymer method: Modeling of macromolecules and aggregates with branching and crosslinking, formed in random processes, Discrete Dynamics in Nature and Society Volume 2 (1998), Issue 3, Pages 203-208 http://dx.doi.org/10.1155/S1026022698000181.

22. Romm F., Figovsky O. Modeling of Mechanical Properties of Polymeric Systems with Branching/Crosslinking, Particularly Their Mechanical Resistance and Stability. Macromolecular Theory and Simulations Volume 11, Issue 1, pages 93-101, January 2002.

23. Romm F., Karcheusky V., Figousky O. Combined Monte Carlo/thermodynamic model of formation of microporous aggregate structure like silica from quaternary ammonium silicate solutions. Journal of Surfactants and Detergents(IF 1.515), 2000, Vol.3 (4), pp. 475-481 Springer. http://onlinelibrary.wiley.com/doi/10.1002/1521-3919\% 2820020101\% 2911:1\% 3C93::AIDMATS93\% 3E3.0.CO;2-F/abstract.

24. Ponomarenko A.T., Figovsky O.L., Shevchenko V.G. Multifunctional Polymer Composites for «Intellectual» Structures: Present State, Problems, Future. Journal Advanced Materials Research, 2008, Vol.740 (47), pp. 81-84, Trans Tech. 
25. Фиговский О.Л., Бейлин Д.А., Поноларев А.Н. Успехи применения нанотехнологий в строительных материалах // Нанотехнологии в строительстве. 2012. - Том 4, №3. - С. 6-21.

26. Кудрявиев П.Г., Фиговский О.Л. Наноструктурированные материалы, получение и применение в строительстве // Нанотехнологии в строительстве. 2014. - Tом 6, № 6. - c. 27-45. - DOI: dx.doi.org/10.15828/2075-8545-2014-66-27-45.

27. Кудрявиев П.Г., Фиговский О.Л. Квазигомогенное приближение для описания свойств дисперсных систем. Основные подходы к моделированию процессов отверждения в нанодисперсных силикатных системах. Часть I. Статистический полимерный метод // Нанотехнологии в строительстве. - 2015. - Том 7 , № 1. - C. 29-54. - DOI: dx.doi.org/10.15828/2075-8545-2015-7-1-29-54.

\section{УВАЖАЕМЫЕ КОЛЛЕГИ!}

ПРИ ИСПОЛЬЗОВАНИИ МАТЕРИАЛА ДАННОЙ СТАТЬИ

ПРОСИМ ДЕЛАТЬ БИБЛИОГРАФИЧЕСКУЮ ССЫЛКУ НА НЕЁ:

Кудрявиев П.Г., Фиговский О.Л. Квазигомогенное приближение для описания свойств дисперсных систем. Основные подходы к моделированию процессов отверждения в нанодисперсных силикатных системах. Часть II. Процессы отверждения с точки зрения статистической физики / / Нанотехнологии в строительстве. - 2015. - Tом 7, № 2. - C. 62-84. - DOI: dx.doi.org/10.15828/20758545-2015-7-2-62-84.

\section{DEAR COLLEAGUES!}

THE REFERENCE TO THIS PAPER HAS THE FOLLOWING CITATION FORMAT:

Kudryavtsev P.G., Figovsky O.L. Quasi-homogenous approximation for description of the properties of dispersed systems. The basic approaches to model hardening processes in nanodispersed silica systems. Part II. The hardening processes from the standpoint of statistical physics. Nanotehnologii v stroitel'stve = Nanotechnologies in Construction. 2015, Vol. 7, no. 2, pp. 62-84. DOI: dx.doi. org/10.15828/2075-8545-2015-7-2-62-84.

\section{Контакты}

\section{Фиговский Олег Львович figovsky@gmail.com}

